Research Paper

\title{
Yes-Associated Protein (Yap) Is Required for Early Embryonic Development in Zebrafish (Danio Rerio)
}

\author{
Jingying $\mathrm{Hu}^{1 *}$, Shuna Sun ${ }^{2}$, Qiu Jiang1, Shaoyang Sun ${ }^{1}$, Wei Wang${ }^{2}$, Yonghao Gui², Houyan Song ${ }^{\circledR}$ \\ 1. Department of Biochemistry and Molecular Biology, Shanghai Medical School and Key Laboratory of Molecular Medicine, Ministry of \\ Education, Fudan University, Shanghai 200032, PR China. \\ 2. Children's Hospital, Fudan University, Shanghai 200032, PR China. \\ * These authors contributed equally to this work.
}

$\triangle$ Corresponding author: Houyan Song, Department of Biochemistry and Molecular Biology, Shanghai Medical School and Key Laboratory of Molecular Medicine, Ministry of Education, Fudan University, Shanghai 200032, PR China.

() Ivyspring International Publisher. This is an open-access article distributed under the terms of the Creative Commons License (http://creativecommons.org/ licenses/by-nc-nd/3.0/). Reproduction is permitted for personal, noncommercial use, provided that the article is in whole, unmodified, and properly cited.

Received: 2012.07.17; Accepted: 2013.02.26; Published: 2013.03.06

\begin{abstract}
The hippo (Hpo) signaling pathway plays a critical role in regulation of organ size. The kinase cascade ultimately antagonizes the transcriptional co-activator Yki/YAP, which is a key regulator of cell proliferation and apoptosis. In this study, we performed a knocking down study using antisense morpholino (MO) reagents and found that zebrafish YAP, a key transcriptional co-activator of Hpo pathway, plays a critical role in early embryonic development. At the cellular level, yap inhibition increases apoptosis and decreases cell proliferation. Reduction of yap function severely delays several developmental events, including gastrulation, cardiogenesis and hematopoiesis. Knockdown of yap showed some evidence of ventralization, including reduction of dorsally expressed marker goosecoid (gsc), expansion of ventral marker gata2, disruption of the somites, and reduction in head size. Finally, we performed a preliminary analysis with real-time polymerase chain reaction (qPCR) for the candidate targets of zebrafish Hpo pathway. In conclusion, our results revealed that zebrafish yap coordinately regulates cell proliferation and apoptosis and is required for dorsoventral axis formation, gastrulation, cardiogenesis, hematopoiesis, and somitogenesis.
\end{abstract}

Key words: Yes-Associated Protein, Yap, Zebrafish

\section{Introduction}

The Hpo pathway, a novel signaling pathway defined in drosophila, is extremely powerful in the regulation of growth and the suppression of tumors because of its ability to simultaneously inhibit cell proliferation and promote apoptosis [1-4]. Activation of the pathway induces a kinase cascade in which the Ste20-type kinase Hpo forms a complex with the WW-domain-containing adaptor protein Salvador (Sav). This complex then phosphorylates and activates the NDR family kinase Warts (Wts). After Wts binds to the Mob family protein Mats, it phosphorylates and inactivates the transcriptional co-activator Yorkie (Yki), which binds to the YEAD/TEF factor Scalloped
(Sd) and other possible transcription factors to regulate the transcription of cell- cycle and cell-death regulators such as cyclin $E$ and diap $1[5,6]$. Like drosophila studies, genetic and cell culture studies in mammalian cells have also linked the Hpo kinase cascade to the phosphorylation of YAP/TAZ, to two mammalian homologs of $\mathrm{Yki}$, (transcriptional co-activator with PDZ-binding motif; also known as WWTR1), and to the regulation of growth [7-13].

The ease of creating genetic mosaics in drosophila has exactly circumvented the hindrance of early lethality following gene depletion in the Hpo signal transduction pathway [4]. However, such efforts 
cannot lead to complete understanding of its in vivo function in early embryonic development. Because of its semitransparent body, genetics and ease of manipulation, zebrafish are considered an ideal vertebrate model for the study of early development and cancer-related genes [14, 15]. Increasing amount of evidence suggest that the Hpo pathway might be evolutionarily conserved in zebrafish [16-20]. Bioinformatics analyses have indicated a considerable amount of evolutionary conservation between zebrafish and other species in the sequences, domains and motifs of several Hpo pathway components, including YAP/TAZ, LATS, Mats, and Fat [16-22]. Mats1, which regulates cell proliferation, apoptosis, and growth, is evolutionary conserved between zebrafish and drosophila [17]. Depletion of zebrafish yap ameliorates the changes in pronephros development caused by Fat1 knockdown [18]. To further elucidate the effects of the components of the hippo signaling pathway on early embryonic development, we investigated the role of yap in the zebrafish embryogenesis. We found that zebrafish yap regulates cell proliferation and survival and that it is required for normal formation of the dorsoventral axis.

\section{Results}

\section{Embryos depleted of Yap exhibit a phenotype of delayed general development}

To determine the role of yap during zebrafish embryogenesis, a translation-blocking morpholino (yap-MO) and a splicing-blocking morpholino (yap-MO(S)) were designed to knock down yap expression (Fig. 1A). The efficacy and specificity of yap-MO was recently confirmed [19]. We performed RT-PCR to demonstrate the activity of yap-MO(S). Our result showed that yap mRNA splicing was completely disrupted in embryos injected with $20 \mathrm{ng}$ of the yap-MO(S). They either lacked exon2 or had an additional $71 \mathrm{bp}$ fragment in intron2 (Fig. 1B). To produce the same phenotype, we used a much higher dose of yap-MO(S) than yap-MO. We also confirmed the knockdown of endogenous Yap protein by Western blotting (Fig. 1C).

Almost all yap-MO-injected embryos showed a phenotype of delayed development, defined by comparison to age-matched control embryos (Fig. $2 \mathrm{~A}-\mathrm{D}$ and $\left.\mathrm{A}^{\prime}-\mathrm{D}^{\prime}\right)$. At $24 \mathrm{hpf}, 51 \%$ of these abnormal yap-MO morphants (2-5 ng of yap-MO per embryo) showed moderate deviation from the morphological norm (Fig. 1D-c, $\mathrm{c}^{\prime}$, and E), 26\% with mild morphological abnormality (Fig. 1D-b, b', and E), and the rest $23 \%$ showed severe morphological deviations (Fig. 1D-d, d', and E). Mild morphants has indistinct heads and tails that bent toward the abdomen, moderate morphants had short bodies and curved trunks, and severe morphants had small trunks and completely indistinct bodies. Mortality was calculated at $48 \mathrm{hpf}$ (Fig. 1E). No death was detected in the mild group $(0 / 24)$. There was a $52 \%$ death rate in the moderate group $(25 / 48)$ and a $64 \%$ death rate in the severe group (14/22). This effect was found to be MO-concentration-dependent (Table 1). Previous work has shown that embryos with reduced Yap exhibit marked increase in cell death of brain at $24 \mathrm{hpf}$ [19]. In this study, we examined yap-MO-injected embryos at multiple time points during the first $24 \mathrm{~h}$ of development and compared them with their wild-type siblings (Fig. 2). At $6 \mathrm{hpf}$, when con-MO-injected or wild-type siblings reached the $60 \%$-epiboly stage, the yap morphants had only developed to about the $50 \%$-epiboly stage. The germ ring appeared thicker than that of wild-type (Fig. 2A and $\left.\mathrm{A}^{\prime}\right)$. At $10 \mathrm{hpf}$. The epiboly of siblings injected with con-MO was already complete but yap morphants showed only $60-80 \%$ epiboly (Fig. $2 \mathrm{~B}$ and $\mathrm{B}^{\prime}$ ). By $18 \mathrm{hpf}$, the yolk extension of con-MO-injected siblings was clearly delimited from the yolk ball as the tail had straightened out, but the yap morphants were developmentally equivalent to wild-type or control embryos at about $14 \mathrm{hpf}$ (Fig. 2C and $\mathrm{C}^{\prime}$ ). At $24 \mathrm{hpf}$, yap-MO-injected embryos displayed a shortened body axis and notable opacity in the head (Fig. 2D and $D^{\prime}$ ).

Table I. Phenotypes observed in subjects exposed to different concentrations of yap-MO.

\begin{tabular}{|c|c|c|c|c|c|c|}
\hline & \multirow[t]{2}{*}{$n$} & \multicolumn{5}{|l|}{ Phenotype } \\
\hline & & Normal & Mild & Moderate & Severe & Number of deaths at $48 \mathrm{hpf}$ \\
\hline con-MO & 100 & $96(96.0 \%)$ & $4(4.0 \%)$ & $0(0.0 \%)$ & $0(0.0 \%)$ & $2(2.00 \%)$ \\
\hline $0.25-1 \mathrm{ng}$ yap-MO & 102 & $26(25.5 \%)$ & $55(53.9 \%)$ & $20(19.6 \%)$ & $1(1 \%)$ & $11(10.78 \%)$ \\
\hline $2-5 \mathrm{ng}$ yap-MO & 99 & $0(0.0 \%)$ & $23(23.2 \%)$ & $51(51.5 \%)$ & $25(25.3 \%)$ & $43(43.43 \%)$ \\
\hline $10-15$ ng yap-MO & 101 & $0(0.0 \%)$ & $2(2.0 \%)$ & $29(28.7 \%)$ & $70(69.3 \%)$ & $61(60.39 \%)$ \\
\hline
\end{tabular}




\section{A Splice blocking:}

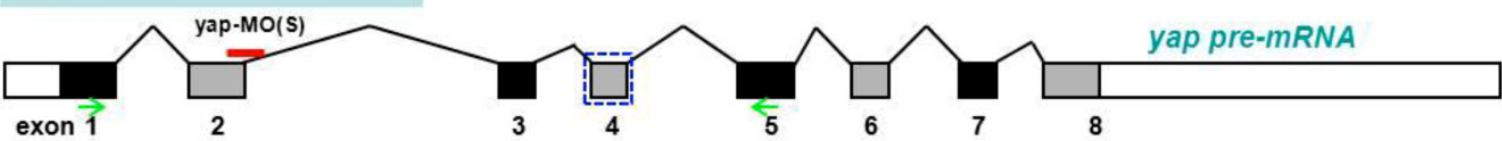

\section{Translation blocking:}

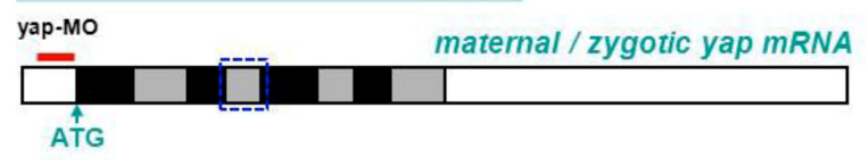

B
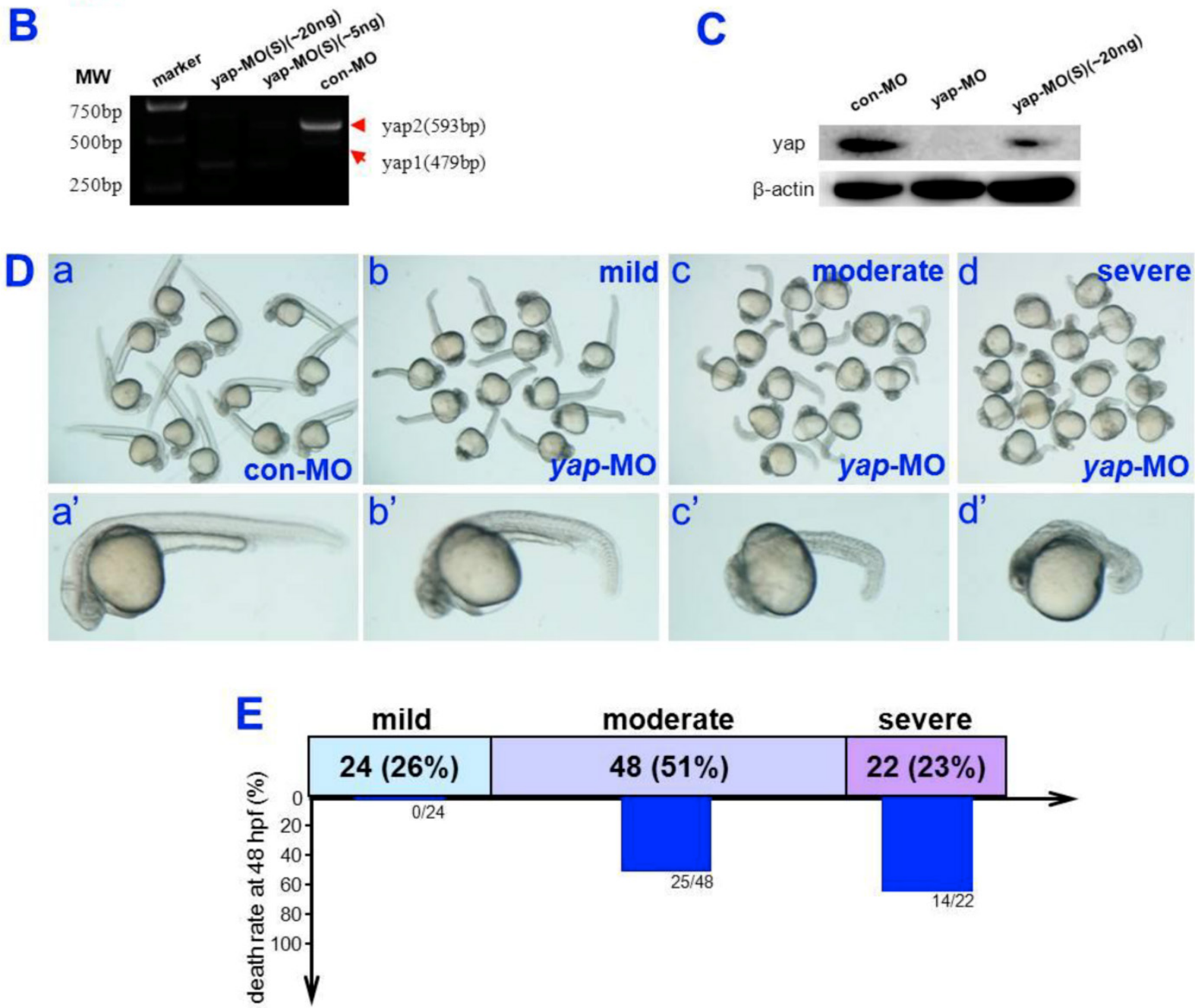

Fig I. Analysis of yap-MOs and yap morphant phenotype. A) Structure of zebrafish yap pre-mRNA (up) and mature mRNA (down). The black lines represent introns, the black rectangles represent exons, and the white rectangles represent untranslated regions (UTR). yap-MO(S) binds to the exon2-intron2 boundary and yap-MO binds to the 5'UTR near the ATG site. The blue rectangular dashed lines represent the WW-domain, which has here been omitted. B) RT-PCR analysis of yap-MO(S). RT-PCR was performed with a pair of primers (green arrows in (A), Table I). C) Western blot analysis of yap-MO and yap-MO(S). Total proteins were extracted from 24 hpf zebrafish embryos. D) Bright-field images of 24 hpf embryos injected with con-MO (2-5 ng/embryo) (a and a') and yap-MO (2-5 ng/embryo) (b-d, b'-d'). At 24 hpf, yap morphants was divided into three groups according to their phenotypes: wild (b and b'), moderate (c and $\left.c^{\prime}\right)$ and severe $\left(d\right.$ and $\left.d^{\prime}\right)$. D) The horizontal bars show the distribution of phenotypes observed in (C), and the vertical bars represent the death rate of each group before $48 \mathrm{hpf}$. 


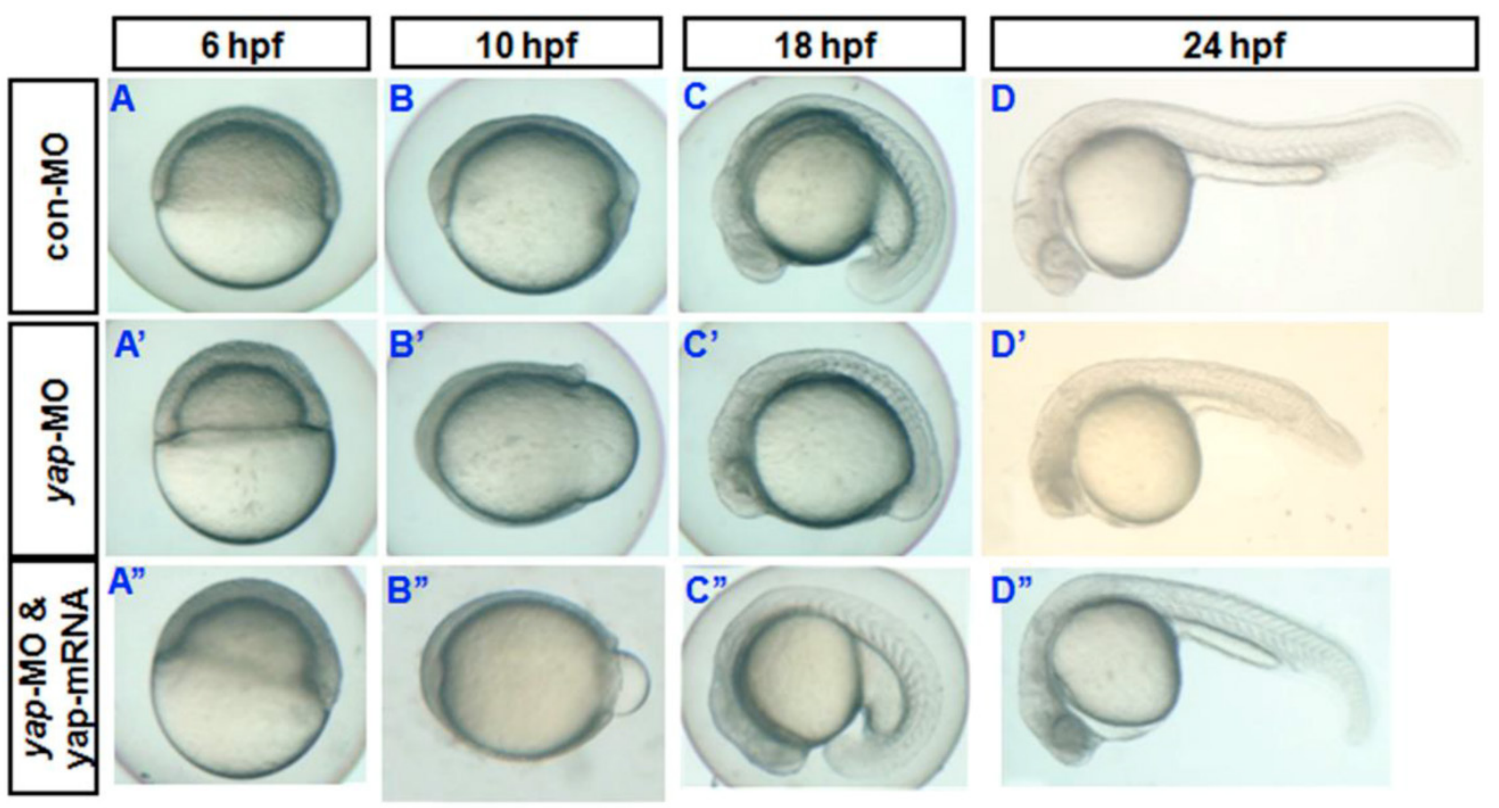

Fig 2. Phenotypes of yap morphant and rescued phenotype. A-D) and $A^{\prime}-D^{\prime}$ ) Bright-field images show yap morphants, all of which exhibited developmental delay. A"-D") This phenotype could be partially rescued after injection of synthesized yap-mRNA.

\section{Both zyapl and zyap2 partially rescued the phenotype of yap morphants}

During sequencing of the expression vector of synthetic yap-mRNA, we found a splicing variant that encodes zebradfish Yap with the first WW-domain. Yap with two WW-domains, known preciously as zebrafish Yap, is here designated as Yap2 and the new Yap, which has only one WW-domain, is here designated as Yap1. Yap1 differs from Yap 2 in that it lacks the WW domain encoded on fourth exon. Yap2 may be a splicing variant (Supplementary Material: Fig. S1). The fourth exon is downstream of and far away from the target region of yap- $\mathrm{MO}(\mathrm{S})$. This is why yap- $\mathrm{MO}(\mathrm{S})$ can disrupt normal splicing (Fig. 1A, B). We injected zebrafish yap1 (zyap1) and yap2 (zyap2) mRNA, both lacking the targeted 5'-UTR, into different individuals. The phenotypes of the resulting yap MO morphants were partially rescued by con injection of yap mRNA (Fig. 2A"- D"). We counted the number of both moderate and severe embryo in each group at $24 \mathrm{hpf}$ and found no obvious differences between yap1 and yap2 with respect to rescue (Fig. 3).

\section{Both cell survival and proliferation are defec- tive in yap morphant embryos}

Yki/YAP is a potent promoter of cell survival and proliferation in drosophila and a variety of cultured mammalian cells [1-4, 7-12], and is inhibited by Hpo signaling. To determine whether knocking down of yap increases the rate of apoptosis in developing vertebrate embryos, a TUNEL assay was performed during the first $24 \mathrm{hpf}$ (Fig. 4A-D and $\mathrm{A}^{\prime}-\mathrm{D}^{\prime}$ ). Both the control embryos and yap morphant embryos showed no or few TUNEL-positive signaling at 6 and $10 \mathrm{hpf}$ (Fig. 4A, A', B, and B'). This may be because apoptotic cell death rarely occurs before $10 \mathrm{hpf}$. The yap-MO-injected embryos clearly exhibited increased apoptosis at 18 and $24 \mathrm{hpf}$, mainly in the head and caudal parts (Fig. 4C, C', D, and $D^{\prime}$ ). In this way, knockdown of yap was found to result in increased cell death during embryonic development.

Then we determined whether cell proliferation in yap morphant embryos was defective. We used phosphohistone $\mathrm{H} 3$ (PH3) antibody staining to mark M-phase cells (Fig. 4E-H and $\mathrm{E}^{\prime}-\mathrm{H}^{\prime}$ ). At earlier stages $(6,10$, and $18 \mathrm{hpf})$, the yap morphant embryos showed fewer mitotic cells than control or wild-type siblings (Fig. 4E,E', F, F', G, and $\mathrm{G}^{\prime}$ ). However, at $24 \mathrm{hpf}$, no significant difference in the $\mathrm{PH} 3$ staining was found between yap morphants and control embryos (Fig. $4 \mathrm{H}$ and $\mathrm{H}^{\prime}$ ). This is consistent with the results of our fluorescence-activated cell sorting (FACS) analysis (data not shown).

\section{Disrupted dorsoventral patterning in yap-depleted embryos}

To determine whether yap inhibition affects markers of dorsoventral patterning during early stages of development, we examined the expression of various ventral and dorsal markers during early and late gastrula stages. There was no obvious dif- 
ference in spatial expression of the ventral marker bmp $2 b$ between yap morphant and control embryos (Fig. 5A and $\mathrm{A}^{\prime}$ ) [23], although there appeared to be a clear overall delay in the development of age-matched morphants relative to control and wild-type siblings. Defective epiboly movement during gastrulation may be the direct cause of the delayed development and similar spatial expression. The same may be true of the absence of dorsal markers no tail (ntl) (Fig. 5D, D',
$\mathrm{E}$, and $\mathrm{E}^{\prime}$ ) and the presence of foxA2 (Fig. 5F and $\mathrm{F}^{\prime}$ ) [24-26]. However, the control expression profiles of gsc and gata2 during gastrulation showed reduced gsc expression in the prechordal plate and increased gata2 expression in the ventral mesoderm rather than a simple delayed pattern (Fig. 5B, B', C, and $C^{\prime}$ ) [27-29]. In total, these data suggested that the reduction of yap disrupted dorsoventral marker expression during zebrafish gastrulation.

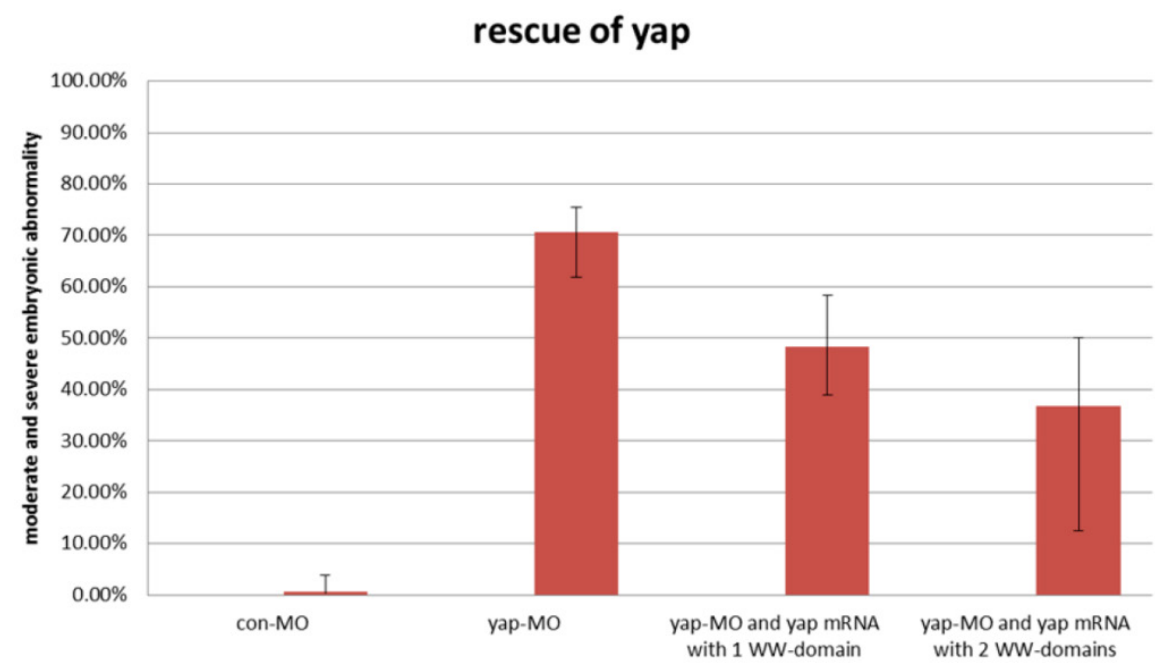

Fig 3. No obvious differences between zyap I and zyap2 with respect to rescue. The percentage of abnormally developed embryos among control embryos, yap morphants, and yap-mRNA rescued morphants. The synthetic yap-mRNAs were found to partially rescue the yap-MO phenotype.

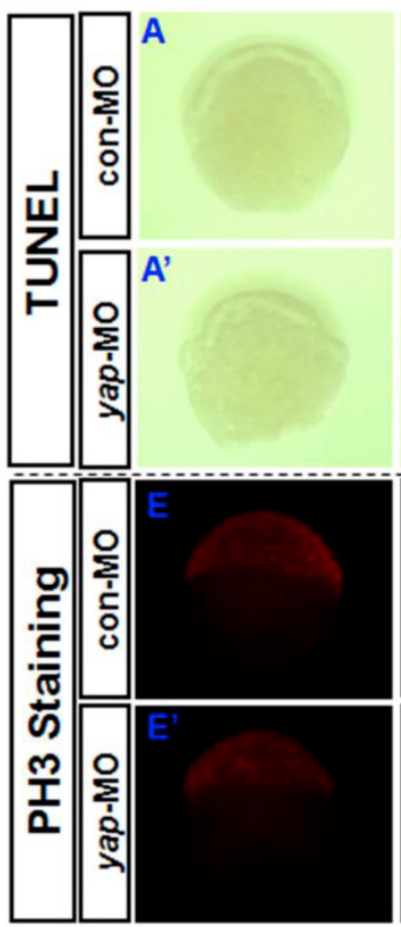

B
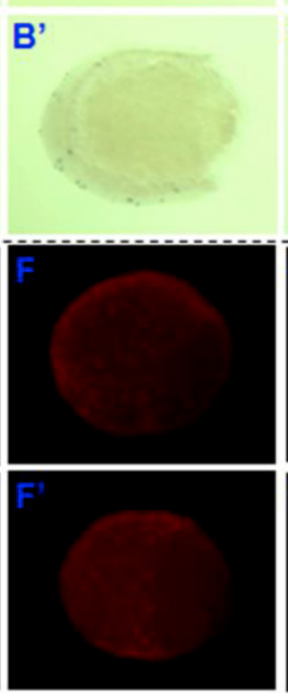

C

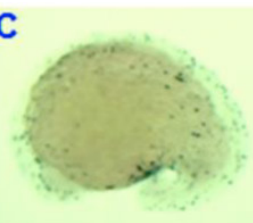

C'
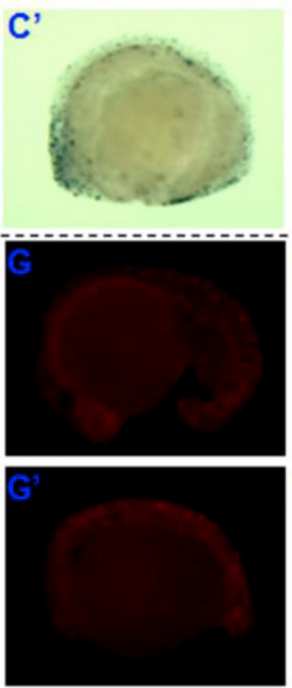

D

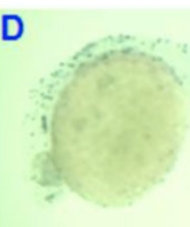

D'
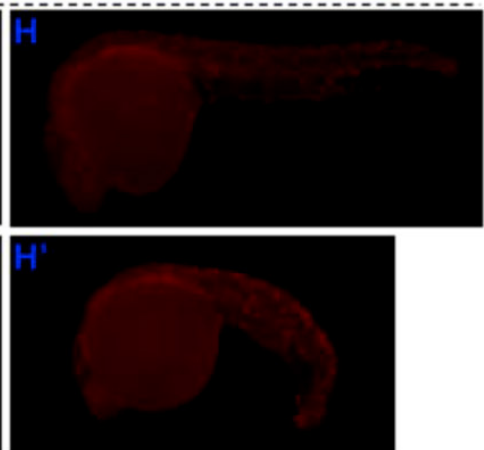

Fig 4. Role of yap in regulation of apoptosis and proliferation during the first $\mathbf{2 4}$ hpf. A-D) and $\left.A^{\prime}-D^{\prime}\right)$ TUNEL staining indicates that knockdown of yap leads to increased cell death in the head and caudal parts. E-H) and $E^{\prime}-H^{\prime}$ ) PH3 antibody staining suggests that yap inhibition results in reduced cell proliferation. 


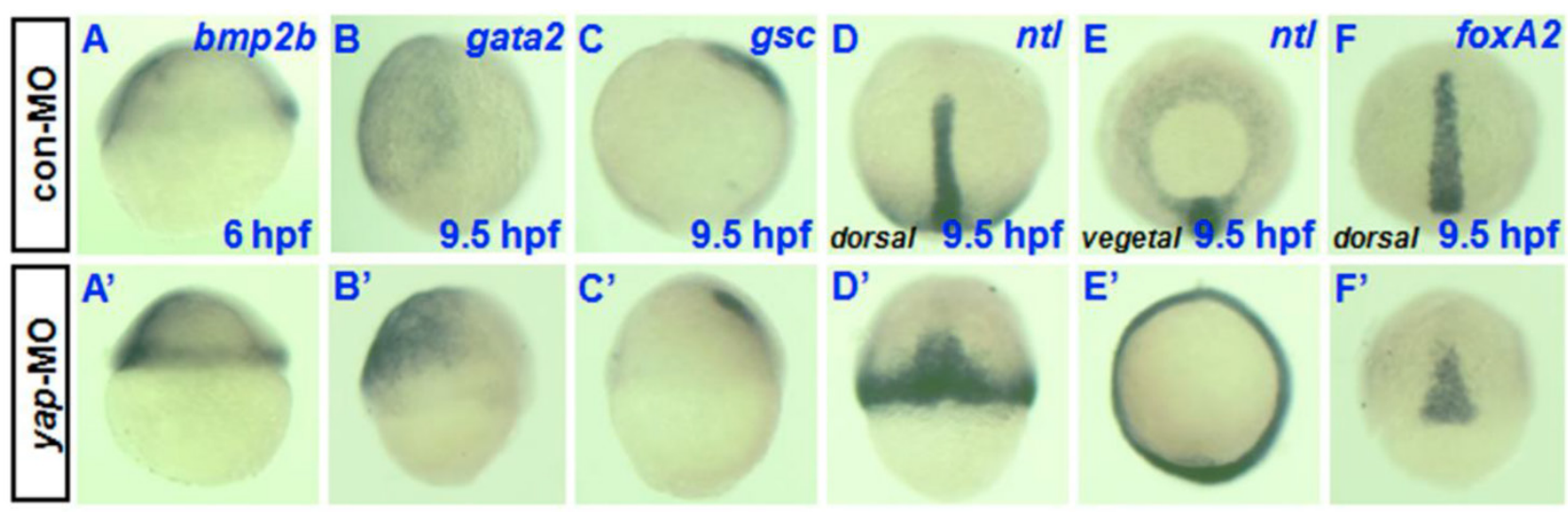

Fig 5. Analysis of dorsoventral markers in yap morphants during gastrulation. $A-F)$ and $\left.A^{\prime}-F^{\prime}\right)$ Whole-mount in situ hybridization of 6-9.5 hpf embryos injected with 2-5 ng con-MO (A-F) and yap-MO (A'-F') for bmp2b (A and $\left.A^{\prime}\right)$, gata2 (B and $\left.B^{\prime}\right)$, gsc (C and $\left.C^{\prime}\right)$, $n t l\left(D, D^{\prime}, E\right.$ and $\left.E^{\prime}\right)$, and foxA2 ( $F$ and $\left.F^{\prime}\right)$. Embryos are shown laterally with ventral to the left ( $A-C$ and $\left.A^{\prime}-C^{\prime}\right)$, dorsally with anterior to the top ( $D, D^{\prime}, F$ and $\left.F^{\prime}\right)$, and vegetally with ventral to the top ( $E$ and $\left.E^{\prime}\right)$.

\section{Effects of zebrafish yap on somitogenesis}

Both the expression pattern of yap during somitogenesis and the shortened body axis of yap-depleted embryos indicate that yap function is critical for somatic development [19]. For this reason, we examined the expression of somatic marker $m y o D$ [30] in control and yap morphants. In situ hybridization showed that the level of $m y o D$ transcription might be up-regulated in yap-depleted embryos relative to control embryos (Fig. 6C and D). To confirm this result, we performed real-time PCR to quantify $m y o D$ expression level at $18 \mathrm{hpf}$. We found a 2-fold increase of myoD mRNA level in yap-morphants compared with control (Fig. 6 I). Despite the delayed onset of somatic development, somitogenesis proceeded faster in yap morphant embryos than that of the control, and yap-depleted embryos and control siblings reached the 18-somite stage at the same time (Fig. 6A-D). titin marks a somatic border in zebrafish embryos [31]. In control embryos, the dorsal and ventral portions of each myotome converge at the point where the horizontal myoseptum forms, and the somites take on a v-shape (Fig. 6E and G). In contrast, the somites in yap-MO-injected embryos appeared U-shaped (Fig. 6F and $\mathrm{H}$ ) [32]. These results indicate that yap morphants have a higher expression level of myoD.

\section{Delayed cardiogenesis and hematopoiesis in yap-MO-injected embryos}

Knockdown of yap causes developmental delay of cardiogenesis and hematopoiesis. In situ hybridization at $24 \mathrm{hpf}$ with cardiac-specific markers $n k x 2.5$ and titin has demonstrated that the heart primordia in yap-MO-injected embryo forms a shallow cone with its apex raised dorsally around the lumen, but the primordia of control embryos had already transformed into a linear tube (Fig. 7A, A', B, and B') [30, 33]. However, there was no observable difference in the timing of blood vessel development between yap morphants and control embryos, as assessed using the vascular marker flk-1 (Fig.7C and C') [34].

Erythrocytes in $30 \mathrm{hpf}$ and $52 \mathrm{hpf}$ embyos were identified by o-dianisdine staining (Fig. 8I), in which the presence of hemoglobin in erythrocytes was shown by a brown color after staining [35]. yap-MO embryos showed no red blood cells (RBC) at $30 \mathrm{hpf}$, but it was significantly recovered at $52 \mathrm{hpf}$. Further analysis of the yap morphant phenotype in $\mathrm{Tg}$ (gata1:EGFP) fish showed a clear delay in hematopoiesis after $28 \mathrm{hpf}$ (Fig.8II). Overall, our data indicate that yap-MO-injected embryos experience delayed cardiogenesis and hematopoiesis.

\section{RT-PCR and real-time PCR analysis for can- didate target genes of the zebrafish Hpo sig- naling pathway}

In drosophila and murine livers, several genes have been shown to be targets of the Hpo pathway. These include the cell-cycle regulator cyclin E (ccne) and cell-death regulator Diap1 [11.36]. To further investigate the mechanism by which the zebrafish Hpo pathway coordinately regulates call proliferation and apoptosis, we searched the updated zebrafish proteome database (http://blast.ncbi.nlm.nih.gov/ Blast.cgi?PROGRAM=blastp\&BLAST_PROGRAMS= blastp\&PAGE_TYPE=BlastSearch\&SHOW_DEFAUL TS=on\&LINK_LOC=blasthome) for candidate target genes of Hpo pathway. Based on sequence similarity, we selected six candidate downstream targets of Hpo pathway to validate using RT-PCR analysis, including cone1, ccne2, cond1, birc2, xiap, and ciapin. We found 
that the transcriptional level of ccne2 was down-regulated in yap-depleted embryos (Fig. 9I). Real-time PCR was used to further confirm the results of RT-PCR (Fig. 9II). In addition, Hpo has been shown to phosphorylate and destabilized Diap1 through non-transcriptional processes $[37,38]$. This is one possible explanation for the lack of marked changes in transcriptional level of these three possible regulators of cell death. In addition, undetected inhibitors of apoptosis may also have been involved in this process.

In mammalian cells, Lats can regulate the activ- ity of TAZ, which shares sequence similarity to YAP and modulates mesenchymal differentiation [13]. In addition, TAZ-deficient zebrafish embryos were found to be defective in bone formation, as in our previous report on the phenotype of yap-MO-injected embryos [39]. For this reason, we determined expression level of TAZ in yap-morphants. We found that the same level of TAZ transcripts was present in yap-depleted embryos and in controls (Fig. 9I), ruling out the possibility that yap-MO produced these RT-PCR results and delayed phenotypes by reducing the level of TAZ.

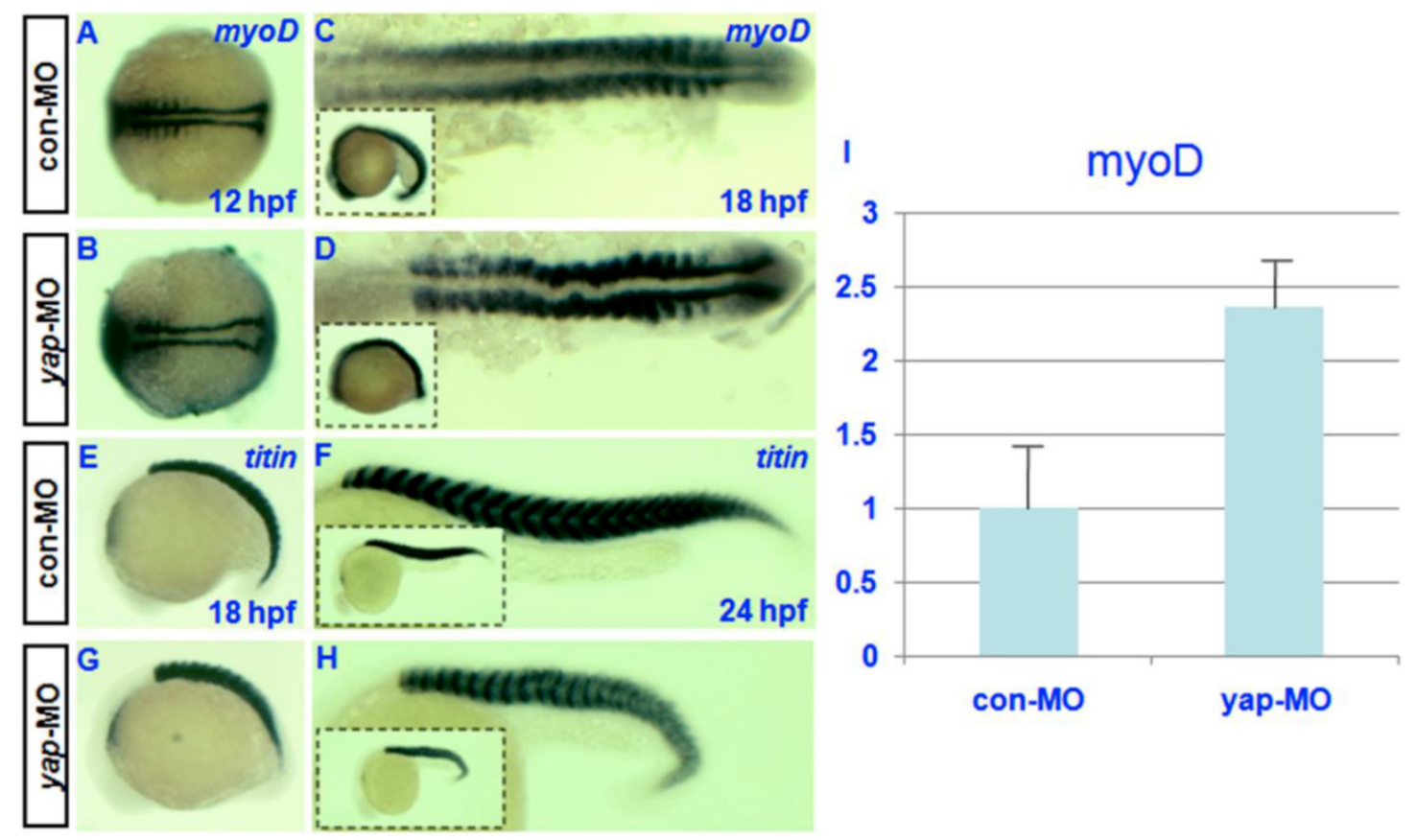

Fig 6. Effect of zebrafish yap on somitogenesis. A-H) Whole-mount in situ hybridization for (A-D) myoD and (E-H) titin at $\mid 2$, 18 , and 24 hpf. A-D) Dorsal view with head to the left. E-H) Lateral view with head to the left. C, D, G, and H) The insets (bottom left corner) show the corresponding whole embryos. I) Real-time PCR result for myoD expression.

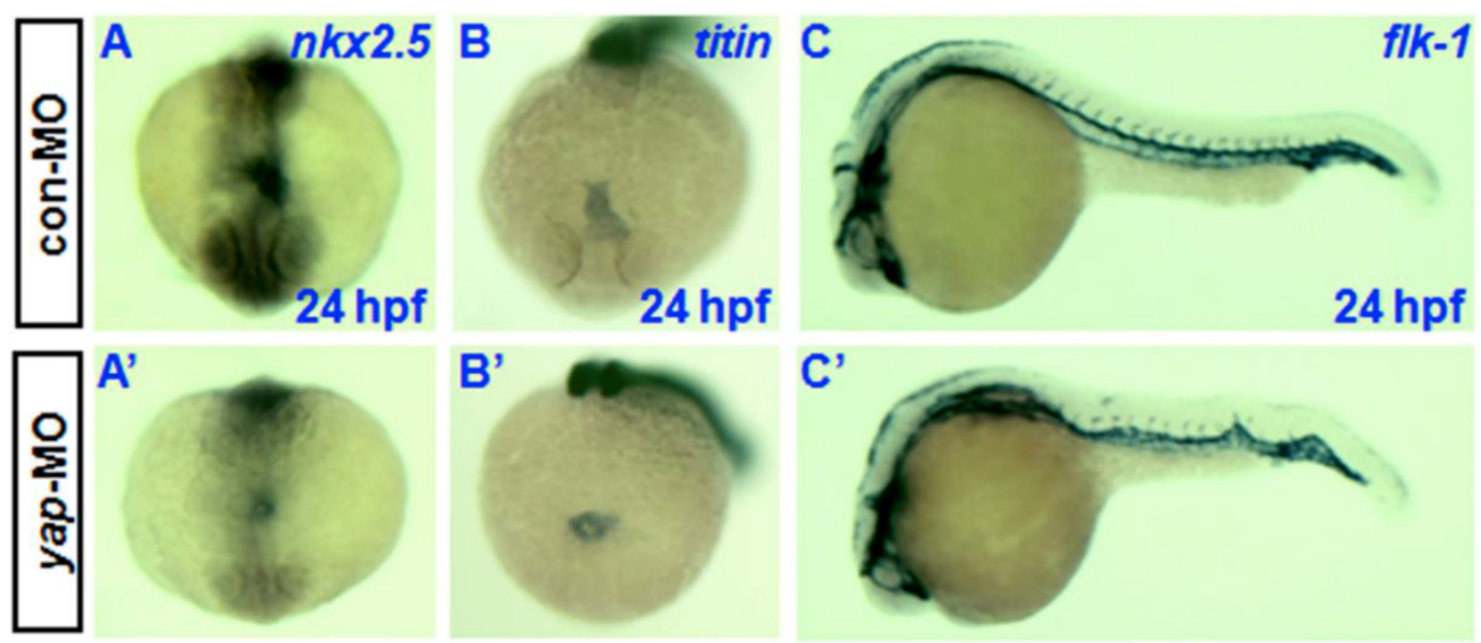

Fig 7. Delayed cardiogenesis and normal vasculogenesis in yap morphants. $A-C$ and $\left.A^{\prime}-C^{\prime}\right)$ Whole-mount in situ hybridization with $n k x 2.5$, titin, and flk-I. $A, A^{\prime}, B$, and $B^{\prime}$ ) Anterior view with dorsal to the top. $C$ and $C^{\prime}$ ) Lateral view with head to the left. 

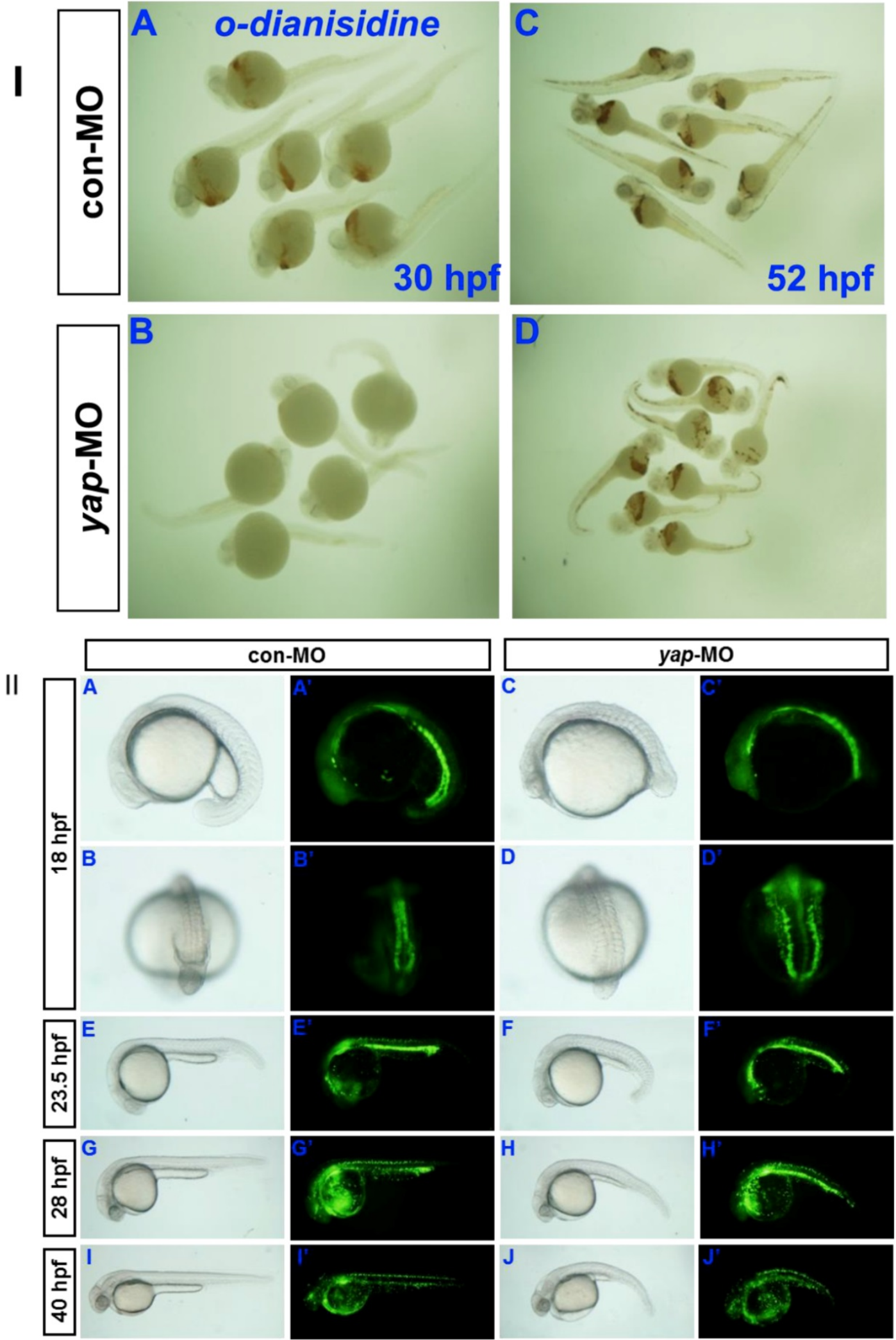

Fig 8. Delayed hematopoiesis in yap morphants. I) O-dianisdine staining shows that yap-MO embryos (B) have no red blood cells (RBC) at $30 \mathrm{hpf}$, with (D) significant recovery by $52 \mathrm{hpf}$. II) (A-J) Bright-field image and ( $\left.\mathrm{A}^{\prime}-\mathrm{J}^{\prime}\right)$ fluorescent image of live $T g($ gatal:EGFP) embryos with (A, A', B, B', E, E', G, G', I, and I') con-MO and (C, C', D, D', F, F', H, H', J, and J') yap-MO. A-J and A'-J') Lateral view with head to the left, except in $B, B^{\prime}, D$, and $D^{\prime}$ (posterior view with dorsal to the top). 

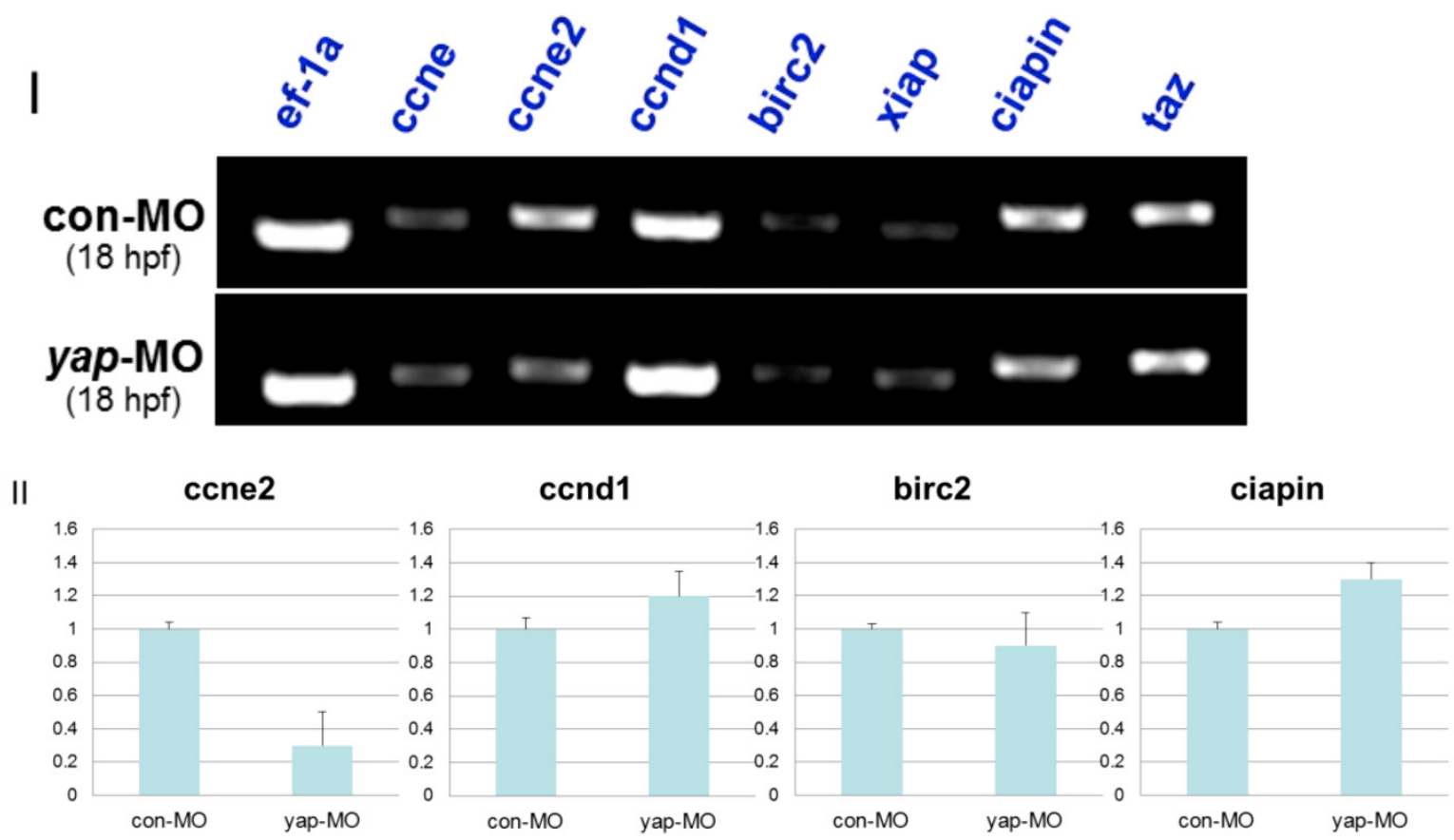

Fig 9. RT-PCR and realtime-PCR analysis for candidate target genes of zebrafish Hpo signaling pathway. I) For each gene (ccne, ccne2, ccndl, birc2, xiap, ciapin, and taz), relative expression based on the RT-PCR is shown. Total mRNA was extracted from I 8 hpf yap-MO and con-MO embryos, respectively. ef-I $a$ was used as a loading control. II) Real-time-PCR result for ccne2, ccndI, birc2, and ciapin.

\section{Discussion}

Previous analysis of the sequence and conservation of the domains of core components of the Hpo pathway have facilitated in-depth investigations on the role of this signaling pathway during zebrafish early embryogenesis [16-20]. Our previous works have shown that yap knockdown caused morphological defects, including small heads, small eyes, and less cartilage in the branchial arches [19]. In this study, the difination of increased cell apoptosis and decreased cell proliferation may be the direct explanation of these morphological defects. Otherwise, we demonstrated that yap-mediated signaling establish specific dorsoventral patterning in zebrafish. Reduction of yap expression was also found to severely delay several developmental events, such as gastrulation, cardiogenesis, and hematopoiesis.

In this study, we tested the efficacy of both the translation-blocking yap-MO [19] and the splice-blocking yap-MO(S) (Fig.1B) by RT-PCR and Western blotting. Our results indicate that both MOs are effective to knock down the endogenous Yap protein, albeit we need to use a higher dose of the yap $M O(S)(20 \mathrm{ng})$. Although we showed a disrupted yap mRNA-splicing in the 20 ng yap $\mathrm{MO}(\mathrm{S})$ group by RT-PCR, there was still a small amount of Yap protein present in the morphants as shown by western blot- ting. It is likely that either our RT-PCR is not sensitive enough, or there are still some maternal Yap proteins present at this stage.

Yap gain of function causes shortened body axis and perturbed somatic and head morphology in both zebrafish and xenopus [20]. Because these late morphologies are similar to the yap knocking down phenotype, it can be difficult to determine whether the rescue is caused by overexpression of yap or knocking down of yap. The ratio of yap MO to yap mRNA needs more experimentation to be determined.

As previously reported, zebrafish Yap (Yap2), like mouse and human YAPs, contains two WW domains. We have identified the one WW domain splicing variant in zebrafish (Yap1). Zebrafish Yaps share significant sequence similarity with their homologues from chickens, mice, and humans (Supplementary Material: Fig. S2). This is especially true of the YAP2s of humans, mice, and zebrafish. Human $\mathrm{YAP}$, which has two WW-domains, is a more potent transcriptional co-activator when compared with YAP with only one WW-domain [40]. In mice, both YAPs have two WW-domains. The first WW-domain has more pronounced affinity for the PPxY sequence, to which it binds more strongly than the second WW-domain does [41]. Although the functions of zebrafish Yap1 and each WW domain of Yap2 are still unknown, the determination of the precise differences 
in signaling among yap splicing variants might be important.

During gastrulation, overall developmental delay increases the difficulty of analyzing defective dorsoventral patterns in yap-MO-injected embryos, but yap knockdown does reduce gsc expression and increase gata2 expression. Confirmation of gsc down-regulation showed that yap-depleted embryos had up-regulated levels of $m y o D$ at $18 \mathrm{hpf}$ with small heads. This is consistent with a previously described pattern in gsc-depleted xenopus embryos [42]. yap-depleted embryos seem to develop a ventralized morphology. During the cell fate specification phase of mouse trophectoderm (TE) development, the Hpo signaling pathway components Lats and Yap increase Tead4 activity, which induces $\mathrm{Cdx} 2$ expression by overcoming Oct3/4-mediated suppression [43]. Maternal zebrafish Spg/Pou2/Oct4 controls the formation of the vertebrate dorsoventral axis and the spg/pou2/oct4 mutant embryos develop a dorsalized morphology [44]. This is in contrast to our ventralized yap morphants, raising the possibility that Yap is required to establish dorsoventral patterning and that it does so by overcoming Spg/Pou2/Oct4-mediated suppression. Further elucidation of the mechanisms by which Yap controls dorsoventral axis formation are required for a more complete understanding of maternal control of embryonic development.

\section{Conclusion}

In conclusion, zebrafish yap is required during early embryonic development. Reduction of yap leads to increased apoptosis, decreased proliferation, disrupted dorsoventral patterns and somitogenesis, as well as delayed cardiogenesis and hematopoiesis. Full identification of zYAP2 and further analysis of yap function are essential to understanding the function of the Hippo signaling pathway in zebrafish embryonic development.

\section{Materials and methods}

\section{Fish care}

The maintenance, breeding, and staging of zebrafish lines ( $\mathrm{AB}$ and Shanghai) were performed as described previously $[45,46]$. The Tg(gata1:EGFP) zebrafish line used in this study has been described elsewhere [47].

\section{Morpholinos and microinjection}

Morpholino (MO) antisense oligonucleotides yap-MO (5'-CTCTTCTTTCTATCCAACAGAAACC3'), yap-MO(S) (5'-GCAACATTAACAACTCACTTTA GGA-3'), and con-MO (5'-CCTCTTACCTCAGTTAC
AATTTATA-3') were obtained from Gene Tools, LLC (U.S.). One- and two-cell stage wildtype and Tg(gata1:EGFP) zebrafish embryos were injected with MOs (2-5 ng/embryo).

\section{Whole-mount TUNEL (terminal deoxynucle- otide transferase mediated dUTP nick-end labeling) staining}

Embryos were fixed in $4 \%$ PFA overnight at $4^{\circ} \mathrm{C}$, then rinsed in PBS before proceeding with whole-mount TUNEL staining using an in situ Cell Death Detection Kit (Roche) according to the manufacturer's instructions.

\section{Whole-mount PH3 antibody staining}

Mitotic cells were stained with a rabbit polyclonal anti phospho-histone H3 (Ser10) antibody (Cell Signaling Technology, Inc.). This was performed almost exactly as described in The Zebrafish Book at a dilution of 1:1000 [46]. The secondary antibody rhodamine (TRITC)-conjugated goat anti-rabbit IgG (Southern Biotech, U.S.) was diluted at 1:200.

\section{Whole-mount in situ hybridization}

Digoxigenin-labeled antisense RNA probes for zebrafish bmp2b, gsc, ntl, foxA2, gata2, myoD, titin, $n k x 2.5$, and $f l k-1$ were synthesized as described in previously published studies. Whole-mount in situ hybridization assay was performed as described [48].

\section{O-dianisidine staining for globin}

Embryos were dechorionated and fixed in $4 \%$ PFA overnight at $4^{\circ} \mathrm{C}$, then rinsed in PBS before proceeding with o-dianisdine staining. Fixed embryos were incubated in the staining buffer for $15 \mathrm{~min}$ in the dark. The staining buffer consisted of $0.6 \mathrm{mg} / \mathrm{ml}$ o-dianisidine (Sigma), $10 \mathrm{mM}$ sodium acetate, $0.65 \%$ hydrogen peroxide, and 40\% (v/v) ethanol.

\section{Reverse transcription-polymerase chain reac- tion (RT-PCR) and quantitative RT-PCR}

Total RNA was extracted from zebrafish embryos using the Trizol reagent (Invitrogen) according to the manufacturer's protocol. Complimentary DNA synthesis was performed using a standard protocol with oligo-dT primers and Moloney murine leukemia virus reverse transcriptase (TaKaRa).

Primers (Table 2) were designed using PRIMER 3 software. PCR conditions were as follows: $95^{\circ} \mathrm{C}$ for 6 min; 30 cycles of $94^{\circ} \mathrm{C}$ for $30 \mathrm{~s} ; 53^{\circ} \mathrm{C}$ for $30 \mathrm{~s}$; and $72^{\circ} \mathrm{C}$ for $45 \mathrm{~s}$; then $72^{\circ} \mathrm{C}$ for $10 \mathrm{~min}$. The products were stored at $4^{\circ} \mathrm{C}$. PCR products were separated on a $1.5 \%$ agarose gel.

Quantitative RT-PCR was performed on the 7500 (ABI) using the SYBR Premix Ex Taq (TaKaRa). 
Measurements were performed in quadruplicate and normalized to the expression levels of Elf1-a. Fold changes values $(X)$ were calculated using the following formula: $X=2-\Delta \Delta C$ t. Bars indicate standard deviations. The PCR cycling conditions were as follows: 40 cycles of pre-denaturation at $95^{\circ} \mathrm{C}$ for $30 \mathrm{~s}$, denaturation at $95^{\circ} \mathrm{C}$ for $5 \mathrm{~s}$, and annealing and extension at $60^{\circ} \mathrm{C}$ for $34 \mathrm{~s}$.

\section{Protein extraction and Western blot analysis}

Total proteins were extracted from zebrafish embryos using RIPA solutions (Cell Signaling Technology, Inc.) according to the manufacturer's proto- col. Protein concentrations were determined using the Bradford assay (BioRad). The proteins were then resolved on SDS/polyacrylamide gels and transferred to polyvinylidene fluoride membranes (Millipore). The membranes were probed with the following primary antibodies: YAP1 (Proteintech) and $\beta$-actin (Cell Signaling Technology, Inc.). After further washes, the membranes were incubated with the appropriate horseradish peroxidase-conjugated secondary antibodies (Proteintech). Blots were developed using ECL (Millipore).

Table 2. Primer sequences.

\begin{tabular}{lllll}
\hline Gene product & Gene bank ID & Upstream primers $\left(5^{\prime}>3^{\prime}\right)$ & Downstream primers $\left(5^{\prime}>3^{\prime}\right)$ & Product size $(\mathrm{bp})$ \\
\hline ccne & NM_130995.1 & cgcagtgctgactgatgaat & actcgagtccccatgacaac & 422 \\
ccne2 & NM_001002075.1 & cacgacaagagcttcatcca & aggtttcctgcgagaactga & 439 \\
ccnd1 & NM_131025.2 & cccaccatctgaccaatacc & gaaaaagcagggagcacttg & 410 \\
birc2 & NM_194395.2 & catcataggggaaggaagca & ccttacaacacgcctggatt & 438 \\
xiap & NM_194396.2 & atggcgaattggaatctgac & atggcgaattggaatctgac & 408 \\
ciapin & NM_001003738.1 & tgcctccagctatgactgtg & tgcatccaaaagagcatctg & 490 \\
taz & NM_001037696.1 & aacagtgggccgtatcactc & tgagagcacaaatccgacag & 415 \\
ef-1a & NM_131263.1 & cttcgtcccaatttcaggat & cagagactcgtggtgcatct & 300 \\
myoD & NM_131262.2 & cgaggtcctgcgaattttag & ctggggtccaagtcttcaaa & 273 \\
yap-sp & NM_001139480.1 & ggttgagaaagctgccagac & tgggaaccttgctttactgg & 583 \\
\hline
\end{tabular}

\section{Supplementary Material}

Fig.S1 - Fig.S2. http://www.ijbs.com/v09p0267s1.pdf

\section{Acknowledgements}

This work was supported by grants from the National Natural Science Foundation of China to Shuna Sun (30901472/H0204), from the National Natural Science Foundation of China to Yuexiang Wang (30600489), and from the Ph.D. Programs Foundation of the Ministry of Education of China to Shuna Sun (200802461111). We would like to thank Prof. Tingxi Liu for his very generous gift of $\mathrm{Tg}$ (gata1: EGFP) zebrafish. We would also like to thank Prof. Lei Zhang, Drs. Jian Yang and Dong Liu for their helpful advice, to thank Dr. Hui Xu for his advice on written English and the other members of Song and Tang's lab for providing technical assistance.

\section{Authers' contributions}

$\mathrm{JyH}, \mathrm{QJ}$, and SnS conceived and designed the experiments. JyH, QJ, and SyS performed the experiments. SnS, WW, and YhG contributed reagents and materials. JyH and $\mathrm{SnS}$ wrote the manuscript. HyS oversaw the experiments and helped to write the manuscript. All authors have read and approved the final manuscript.

\section{Competing Interests}

The authors have declared that no competing interest exists.

\section{References}

1. Edgar BA. From cell structure to transcription: Hippo forges a new path. Cell. 2006; 124: 267- 273.

2. Harvey K, Tapon N. The Salvador-Warts-Hippo pathway - an emerging tumour-suppressor network. Nat Rev Cancer. 2007; 7: 182- 191.

3. Pan D. Hippo signaling in organ size control. Genes Dev. 2007; 21: 886897.

4. Saucedo LJ, Edgar BA. Filling out the Hippo pathway. Nat Rev Mol Cell Biol. 2007; 8: 613- 621.

5. Wu S, Liu Y, Zheng Y, et al. The TEAD/TEF family protein Scalloped mediates transcriptional output of the Hippo growth-regulatory pathway. Dev Cell. 2008; 14: 388- 398.

6. Zhang L, Ren F, Zhang Q, et al. The TEAD/TEF family of transcription factor Scalloped mediates Hippo signaling in organ size control. Dev Cell. 2008; 14: 377- 387.

7. Hao Y, Chun A, Cheung K, et al. Tumor suppressor LATS1 is a negative regulator of oncogene YAP. J Biol Chem. 2008; 283: 5496- 5509.

8. Lee JH, Kim TS, Yang TH, et al. A crucial role of WW45 in developing epithelial tissues in the mouse. EMBO J. 2008; 27: 1231- 1242.

9. Zhang J, Smolen GA, Haber DA. Negative regulation of YAP by LATS1 underscores evolutionary conservation of the Drosophila Hippo pathway. Cancer Res. 2008; 68: 2789- 2794. 
10. Zhao B, Wei X, Li W, et al. Inactivation of YAP oncoprotein by the Hippo pathway is involved in cell contact inhibition and tissue growth control. Genes Dev. 2007; 21: 2747- 2761.

11. Dong J, Feldmann G, Huang J, et al. Elucidation of a universal size-control mechanism in Drosophila and mammals. Cell. 2007; 130: 1120- 1133 .

12. Camargo FD, Gokhale S, Johnnidis JB, et al. YAP1 increases organ size and expands undifferentiated progenitor cells. Curr Biol. 2007; 17: 20542060.

13. Lei QY, Zhang $\mathrm{H}$, Zhao B, et al. TAZ promotes cell proliferation and epithelial-mesenchymal transition and is inhibited by the hippo pathway. Mol Cell Biol. 2008; 28: 2426- 2436.

14. Amatruda JF, Shepard JL, Stern HM, et al. Zebrafish as a cancer model system. Cancer Cell. 2002; 1: 229- 231.

15. Stern HM, Zon LI. Cancer genetics and drug discovery in the zebrafish. Nat Rev Cancer. 2003; 3: 533- 539.

16. Wang K, Degerny C, Xu M, et al. YAP, TAZ, and Yorkie: a conserved family of signal-responsive transcriptional coregulators in animal development and human disease. Biochem Cell Biol. 2009; 87: 77- 91.

17. Yuan Y, Lin S, Zhu Z, et al. The mob as tumor suppressor (mats1) gene is required for growth control in developing zebrafish embryos. Int J Dev Biol. 2009; 53: 525- 533.

18. Skouloudaki K, Puetz M, Simons M, et al. Scribble participates in Hippo signaling and is required for normal zebrafish pronephros development. Proc Natl Acad Sci. 2009; 106: 8579- 8584.

19. Jiang $\mathrm{Q}$, Liu D, Gong $\mathrm{Y}$, et al. yap is required for the development of brain, eyes, and neural crest in zebrafish. Biochem Biophys Res Commun. 2009; 384: 114- 119.

20. Gee ST, Milgram SL, Kramer Kl, et al. Yes-associated protein 65 (YAP) expands neural progenitors ans regulates Pax3 expression in the neural plate border zone. Plos ONE. 2011; 6: e20309

21. Nejigane S, Haramoto Y, Okuno M, et al. The transcriptional coactivators Yap and TAZ are expressed during early Xenopus development. Int J Dev Biol. 2011; 55: 121- 126.

22. Chen $\mathrm{CH}$, Sun $\mathrm{YH}$, Pei DS, et al. Comparative expression of zebrafish Lats1 and Lats2 and their implication in gastrulation movements. Dev Dyn. 2009; 238: 2850- 2859.

23. Xiong B, Rui Y, Zhang M, et al. Tob1 controls dorsal development of zebrafish embryos by antagonizing maternal beta-catenin transcriptional activity. Dev Cell. 2006; 11: 225- 238.

24. Schulte-Merker S, van Eeden FJ, Halpern ME, et al. no tail (ntl) is the zebrafish homologue of the mouse T (Brachyury) gene. Development. 1994; 120: 1009- 1015.

25. Odenthal J, Nusslein-Volhard C. fork head domain genes in zebrafish. Dev Genes Evol. 1998; 208: 245-258.

26. McFarland KN, Warga RM, Kane DA. Genetic locus half baked is necessary for morphogenesis of the ectoderm. Dev Dyn. 2005; 233: 390-406.

27. Montero JA, Carvalho L, Wilsch-Brauninger M, et al. Shield formation at the onset of zebrafish gastrulation. Development. 2005; 132: 1187- 1198.

28. Inbal A, Topczewski J, Solnica-Krezel L. Targeted gene expression in the zebrafish prechordal plate. Genesis. 2006; 44: 584-588.

29. Read EM, Rodaway AR, Neave B, et al. Evidence for non-axial A/P patterning in the nonneural ectoderm of Xenopus and zebrafish pregastrula embryos. Int J Dev Biol. 1998; 42: 763- 774.

30. Weinberg ES, Allende ML, Kelly CS, et al. Developmental regulation of zebrafish MyoD in wild-type, no tail and spadetail embryos. Development. 1996; 122: 271-280.

31. Xu X, Meiler SE, Zhong TP, et al. Cardiomyopathy in zebrafish due to mutation in an alternatively spliced exon of titin. Nat Genet. 2002; 30: 205- 209.

32. van Eeden FJ, Granato M, Schach $U$, et al. Mutations affecting somite formation and patterning in the zebrafish, Danio rerio. Development. 1996; 123: 153- 164.

33. Yelon D. Cardiac patterning and morphogenesis in zebrafish. Dev Dyn. 2001; 222: 552- 563.

34. Fouquet B, Weinstein BM, Serluca FC, Fishman MC. Vessel patterning in the embryo of the zebrafish: guidance by notochord. Dev Biol. 1997; 183: $37-48$.

35. Chu $\mathrm{CY}$, Cheng $\mathrm{CH}$, Chen GD, et al. The zebrafish erythropoietin: Functional identification and biochemical characterazation. FEBS Lett. 2007; 581: 4265-4271

36. Huang J, Wu S, Barrera J, et al. The Hippo signaling pathway coordinately regulates cell proliferation and apoptosis by inactivating Yorkie, the Drosophila Homolog of YAP. Cell. 2005; 122: 421- 434.
37. Harvey KF, Pfleger CM, Hariharan IK. The Drosophila Mst ortholog, hippo, restricts growth and cell proliferation and promotes apoptosis. Cell. 2003; 114: 457-467.

38. Pantalacci S, Tapon N, Leopold P. The Salvador partner Hippo promotes apoptosis and cell-cycle exit in Drosophila. Nat Cell Biol. 2003; 5: 921927.

39. Hong JH, Hwang ES, McManus MT, et al. TAZ, a transcriptional modulator of mesenchymal stem cell differentiation. Science. 2005; 309: 1074- 1078.

40. Komuro A, Nagai M, Navin NE, et al. WW domain-containing protein YAP associates with ErbB-4 and acts as a co-transcriptional activator for the carboxyl-terminal fragment of ErbB-4 that translocates to the nucleus. J Biol Chem. 2003; 278: 33334- 33341.

41. Yagi R, Chen LF, Shigesada $\mathrm{K}$, et al. A WW domain-containing yes-associated protein (YAP) is a novel transcriptional co-activator. EMBO J. 1999; 18: 2551- 2562.

42. Sander V, Reversade B, De Robertis EM. The opposing homeobox genes Goosecoid and Vent1/2 self-regulate Xenopus patterning. EMBO J. 2007; 26: 2955- 2965.

43. Nishioka N, Inoue $\mathrm{K}$, Adachi $\mathrm{K}$, et al. The Hippo signaling pathway components Lats and Yap pattern Tead4 activity to distinguish mouse trophectoderm from inner cell mass. Dev Cell. 2009; 16: 398- 410.

44. Reim G, Brand M. Maternal control of vertebrate dorsoventral axis formation and epiboly by the POU domain protein Spg/Pou2/Oct4. Development. 2006; 133: 2757- 2770.

45. Kimmel CB, Ballard WW, Kimmel SR, et al. Stages of embryonic development of the zebrafish. Dev Dyn. 1995; 203: 253- 310.

46. Westerfield M. The Zebrafish Book: A Guide for the Laboratory Use of Zebrafish (Danio rerio), 4 edn. Oregon, USA: University of Oregon Press; 2000.

47. Dong $\mathrm{M}, \mathrm{Fu} \mathrm{YF}, \mathrm{Du} \mathrm{TT}$, et al. Heritable and lineage-specific gene knockdown in zebrafish embryo. PLoS One. 2009; 4: e6125.

48. Thisse C, Thisse B. High-resolution in situ hybridization to whole-mount zebrafish embryos. Nat. protoc. 2008;3: $59-69$ 